\title{
Atmospheric monitoring at the site of the Telescopio Nazionale Galileo
}

\author{
Ghedina Adriano ${ }^{a}$, Pedani Marco, and Garcia de Gurtubai Albar \\ Fundacion Galileo Galilei INAF, Rambala J.A. Fernandez Perez, 7, 38712 Breña Baja, S.C.Tenerife, Spain
}

\begin{abstract}
For more than 20 years, the atmosphere above the Telescopio Nazionale Galileo and its surroundings at the Roque de Los Muchachos Observatory have been monitored. From the first instruments installed in the early ' 90 s to characterize and show the quality of the sky at the chosen site (particularly the Automatic Weather Station and the DIMM) we evolved into the actual set up of remote sensing devices. They are as a fundamental aid for the telescope, in particular for its safety and the optimization of the scientific throughput. We will resume here the lessons learned, some results and the new choices for monitoring the atmosphere at the TNG.
\end{abstract}

\section{Introduction}

The Telescopio Nazionale Galileo (TNG) [1] is a $4 \mathrm{~m}$ class telescope sited at the Roque de Los Muchachos Observatory in La Palma, Canary Islands. It is operated by the Fundacion Galileo Galilei, and funded by the Italian Institute of Astrophysics INAF. TNG is an Alt-Az telescope with Ritchey Chrétien optical configuration and 2 Nasmyth stations. It begun scientific observations in 1998 and now, with HARPS-N [6], it is one of the leading telescopes in the search for exoplanets.

Since before the begin of regular observations with the telescope the atmosphere above the TNG site has been equipped and monitored with a seeing monitor $(1994 / 95$ [20,22]) and an automatic weather station (1996 [24]). With time the family of atmospheric monitoring systems has grown with other weather stations (2000), a particle counter (2001), a new DIMM (2011 [8]), and other smaller systems. The main reason for the monitoring has also evolved from the initial basic and fundamental characterization of the atmosphere at the site to the nowadays need of safety for the telescope and optimization of the observing programs. We will review in this paper which instruments have been originally installed and which are now used at the TNG site to monitor the atmosphere with the aim of: protect telescope and observers from dangerous situations, optimize observing time and image quality, complete a database of information which could be useful to derive trends, statistical behaviors and forecast weather and seeing.

\section{Automatic weather stations}

Since 1996 weather parameters are registered thanks to the Galileo Automatic Weather Station (GAWS [24]), a $15 \mathrm{~m}$ high tower with temperatures sensor (at ground and at 2, 5 and $10 \mathrm{~m}$ above it), relative humidity sensors and barometer at $5 \mathrm{~m}$ height and, at the top of the tower, wind speed and direction sensors. Data are recorded and archived every 30 seconds and publicly available at the web page of the TNG [31]. After the GAWS was installed it gave fundamental information on the

\footnotetext{
a e-mail: ghedina@tng.iac.es
}

characterization of the TNG site, in particular on yearly averages of temperatures, winds and relative humidity. Once the telescope begun observations (mid of year 1998) the main purpose of monitoring the atmosphere with the weather station became the safety of the telescope.

While the GAWS is placed in a strategical position for the site characterization [7], because it is windward as far as the main trade winds are concerned and not affected by the presence of buildings and structures of the Observatory, it is on the contrary misplaced as far as the safety of the telescope is concerned. Clouds, which typically come from the East and from the edge of the Caldera, arrive at the telescope way before than being detected by the GAWS. Furthermore every winter the tower becomes a column of ice; the combined effect of ice, wind and sun brakes the weather sensors one by one and it is really difficult to maintain the tower continuously operative (access to replace the broken sensors is by climbing on the tower). We often had to observe without our weather sensors in the bad weather season and rely only on the information given from the meteo web pages of the other telescopes on the mountain. To mitigate the above problems in the year 2000 we set up a Davis weather station on the roof of the rotating dome which is much easier to maintain and has much cheaper spare parts. Begin so close to the building it is not reliable for site characterization and it still is too close to the building to give enough response time in case of bad weather.

Data from both weather stations are first of all used for the safety of the telescope, and this is the reason for measuring a new value every 30 seconds. The Operator during observations is continuously checking the weather parameters, in particular the wind speed and the relative humidity, on which specific limits have been chosen in order to consider the environment safe for the observations. To avoid damages to the structure, optics and electronics at the TNG we decided that observations should not be carried out with a wind speed value of more than $20 \mathrm{~m} / \mathrm{s}$ or a relative humidity higher than $85 \%$. If the limits are reached visual and acoustic alarms are activated and the dome is closed. Also the dew point (depending on $\mathrm{P}, \mathrm{T}$ and $\mathrm{RH}$ ) is calculated and displayed in order to know that there is the risk of condensation on the structure and of 


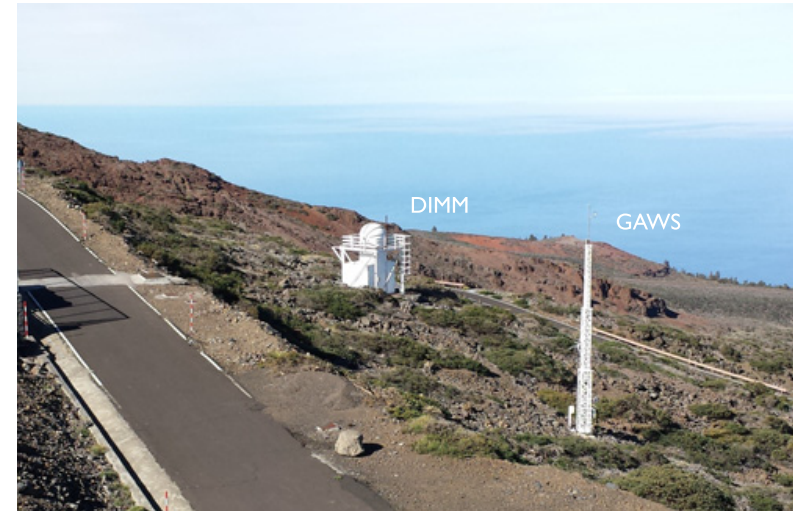

Figure 1. A view from the TNG of the Galileo Automatic Weather Station and of the DIMM.

water drops falling on the optics. To have a better and wider idea of the weather conditions on the mountain the weather stations of the other telescopes are also checked by Telescope Operators during the night; in particular having a look at the other weather stations gives a global view of the meteorological situation of the site (and also lets you know that the web page of our weather station is not frozen). It is not so important when the weather is fine and the sky clear, and only small differences in the measured parameters at the different sites can be observed; it is nonetheless very useful when bad weather is approaching and warnings from the most distant weather stations give enough lag time to take actions at our site.

The air temperature readings recorded during nighttime are also used to calculate the set-point temperature of the air conditioning system for the following day. As soon as the dome is closed at the end of night observations, the air inside the dome is thermally controlled in order to keep the mechanical structure and the optics of the telescope at the calculated temperature. Temperature gradients between air and structure or air and optics produce micro-turbulence which results in image blur (typically known as mirror and dome seeing). So knowing the temperature trend at the end of the night and during the first part of the morning allows to actively adjust the air conditioning system during the rest of the day and minimize the difference in temperature of the telescope with the open air at the moment of opening the dome.

The whole set of weather data are recorded and populate the database we begun in 1996. It is possible to use these data to find correlation with other ground and airborne atmospheric parameters and from these derive weather statistics, trends [13,14], forecasting, etc. [3-5]. It is common also to group the data collected from this and other stations on the mountain and compare this Observatory with other astronomical sites (Paranal for example [17]).

\section{DIMM}

The TNG site has been hosting seeing measurement campaigns since 1994 when a Differential Image Motion Monitor (DIMM[28]) from the Nice Department of Astrophysics was installed by IAC and Italy [22]. Later on, Italy built its own seeing monitor [20] with a dedicated

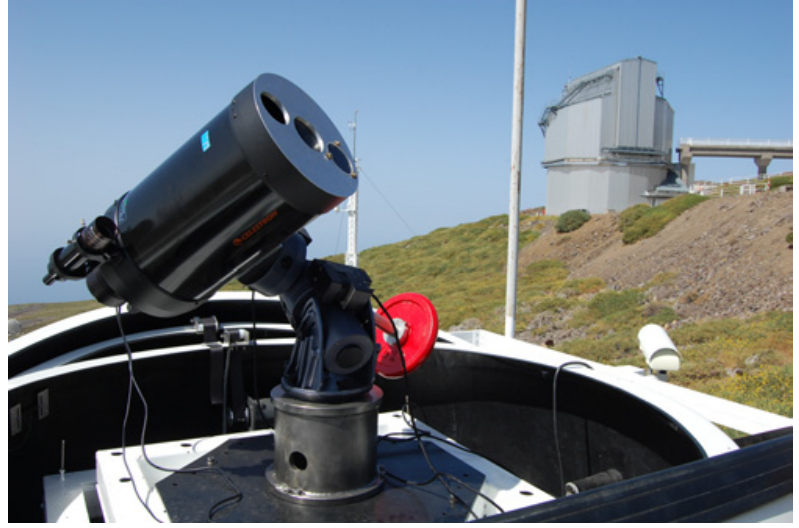

Figure 2. The new DIMM is based on a Celestron 11 CGE-XLT, with 2 pupils with of $80 \mathrm{~mm}$ separated by $198 \mathrm{~mm}$. One of the pupil is covered with a prism with a wedge of 40 arcsecs. The CCD detector is a Sony ICX274AL/AQ of $1600 \times 1200$ pixel, a squared pixel size of $4.4 \mu \mathrm{m}$, and a FoV of $8.11 \times 6.08$ arcmin.

tower [21] and begun regular measurement campaigns independently from the other institutions. After few years of work the original DIMM begun to show the effects of age, in particular on the telescope control and camera systems (motors and electronics): the frequency of failures increased and the lack of support from the builder forced the stop of seeing measurements. A new system (see Fig. 2) still based on DIMM was then funded and built by the TNG staff $[8,9]$ and begun regular observations in march 2011. This is an automatic system which gives a new seeing measurement every 45 seconds based on the statistics of 500 frames of stars. Seeing data are always publicly available on the web page [31].

The seeing campaigns are usually aimed at the characterization of the site and so it was at the beginning also for the TNG site. Similarly to what happened for the weather station, once the telescope is installed and begins observations, the interest in the collected data moves from the night-time (or longer term) behavior of the measured parameters to the instantaneous (every 45 seconds) seeing values, in order to optimize the scientific observations. In particular during service and queuing observing, knowing the value of seeing through the night allows to prioritize programs and increase efficiency, by recalculating exposure times, changing instrument configuration, and improve the overall image quality with the Active Optics. Furthermore the database of seeing values is still being populated with the new values and these data can be helpful in the search of long time trends, of seasonal variations, or of some correlation with wind direction and its speed.

The natural seeing measured by the DIMM during the night gives a fundamental feed-back on the performances of the telescope. A discrepancy between the measured Point Spread Function (PSF) at the telescope and the seeing at the DIMM is usually considered as the result of some turbulent layer within the walls of the dome [27], either close to the surface of the mirror or in the environment, or both. A comparison between the measured seeing and the image quality of the telescope in terms of PSF allows to quantify the amount of seeing induced 


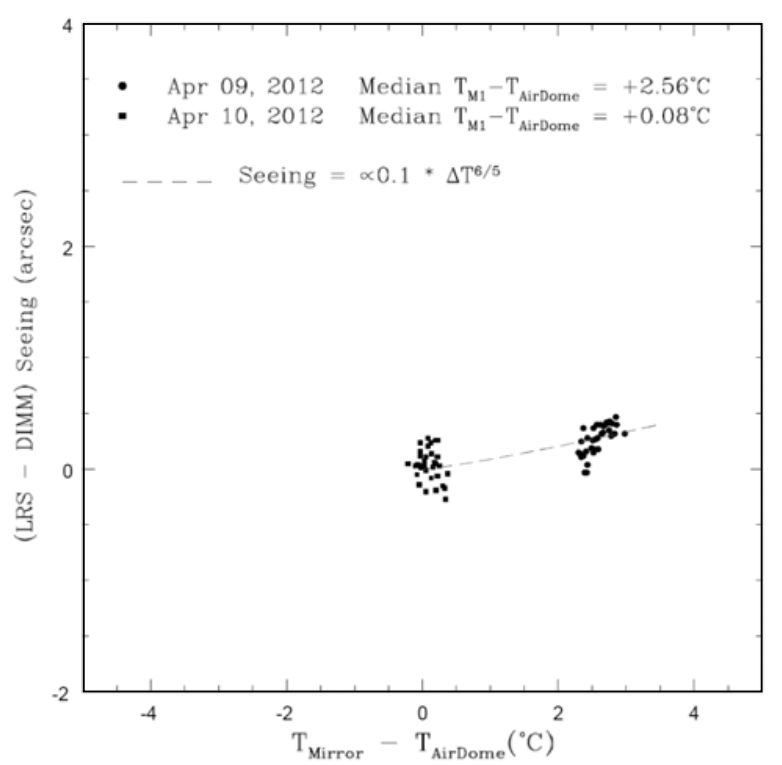

Figure 3. The PSF measured with the instrument LRS at the telescope with respect to the measured natural seeing (on the vertical axis, in arcsec) is related to the temperature difference between the mirror and the air (horizontal axis, in Celsius degrees). Two different nights of measurements are shown with black circles or boxes. From this plot it can be seen that the increase in the telescope PSF with respect to the seeing at the DIMM can be associated to the temperature gradient between a mirror warmer than the surrounding air. The empirical law that could describe this behavior is anyway slightly different from what is found in the literature [27].

by mirror or dome. An important step to remove dome and mirror seeing is to have the air conditioning system tuned up in order to keep the whole structure at the same temperature of the free air entering the dome and minimize temperature gradients between them. As an example, it can be seen in Fig. 3 how the increase in the instrumental PSF with respect to the natural seeing could be associated to the temperature differences between the mirror and the air of the environment. The relationship measured at the TNG is not so strong as reported by Racine [27] and moreover it would be interesting to study the behavior when the mirror is colder than the air. We are shortly going to mount a group of fans on the front and back of the main mirror cell in order to create a laminar flow of air over the mirror. This should wipe away the temperature gradient induced micro-turbulence of the air laying over the optical surface. In doing so we expect to reduce the overall PSF of the telescope and bring it closer to the values of natural seeing.

\section{Particle counter}

Canary Islands, a few hundreds $\mathrm{km}$ west of the African shores, are subject to sand outburst events (called Calima) from the Sahara and Sahel regions [10,12]. We installed at the TNG a portable particle counter in 2001 (abacus TM301, [11,25,26]). The particle counter is mounted on the inside wall of the dome, at the height of the primary mirror, with a pipe going through the wall and sucking the air from the outside. This system was originally designed for clean room environments, and could store only a small amount of data that soon had to be downloaded by an operator through serial cable; sometimes data were lost during the download or overwritten on the counter. We decided to go for an automatic improved system with remote connection and in 2006 this system was replaced by the LasairII 310B, with better performances. Both instruments are from Particle Measuring Systems. The systems measure the concentration of particles in the air, separated in sizes of $0.3,0.5,1.0,3.0,5.0$ and $10 \mu \mathrm{m}$, through back-scattering from a laser diode. Every 2 hours the value of dust concentration in $\mu \mathrm{g} \mathrm{m}^{-3}$ is recorded and publicly available on the TNG web page [31].

Continuous monitoring of dust at the observatory is useful to have a clue on the transparency of the sky and possible extinction [11] and on the particle deposition over the optics (which affects reflectivity and increases emissivity in the NIR) $[15,16,18,19]$. It is not so straightforward to derive the atmospheric extinction from the measured value of dust concentration because, being the latter a local measurement, it does not take into account the whole integrated line of sight and it is not always possible to assume a uniform dust distribution. A direct comparison of the concentration of dust with the measurements of extinction by the Carlsberg Meridian Circle Telescope, as in Fig. 4, shows this problem. Measuring high concentration of dust could be due to a local layer with anyway low values of extinction; on the other side, a high altitude thick layer could absorb and scatter most of the light from the stars but from the measured concentration of dust one could not assume the effect of a Calima outburst. Moreover, in case of a high altitude layer there must be a time delay due to the settling time of dust, and the extinction measured earlier would only be recorded as dust in a later moment.

As far as safety is concerned during and after a Calima outburst there is no documented damage at the Observatory produced by dust over mechanics and electronics (but filters of air conditioning and cabinets' fans must be cleared and changed on a regular basis). The worst effect is the evidence of dust deposition over the optics which (if combined with strong wind) could produce scratches and micro-damages to the reflective coatings if it is not promptly removed. For the above reasons it has not been clearly defined a threshold value for dust concentration above which the observations should be interrupted and the dome closed (as it is for wind speed or $\mathrm{RH}$ ). In principle observations can go ahead uninterrupted but their quality is clearly affected when the dust concentration of $100 \mu \mathrm{g}$ $\mathrm{m}^{-3}$ or more; in this environment the extinction is usually higher than 0.4 magnitudes in the $\mathrm{V}$ band [11], comparable to thick cirri. Calima can also increase the level of the Night Sky Background due to back-scattering of street and city lights [2].

\section{Smaller instruments}

In the framework of trying to monitor the parameters that could affect observations and the safety of the personnel or of the telescope, we decided to include a few 

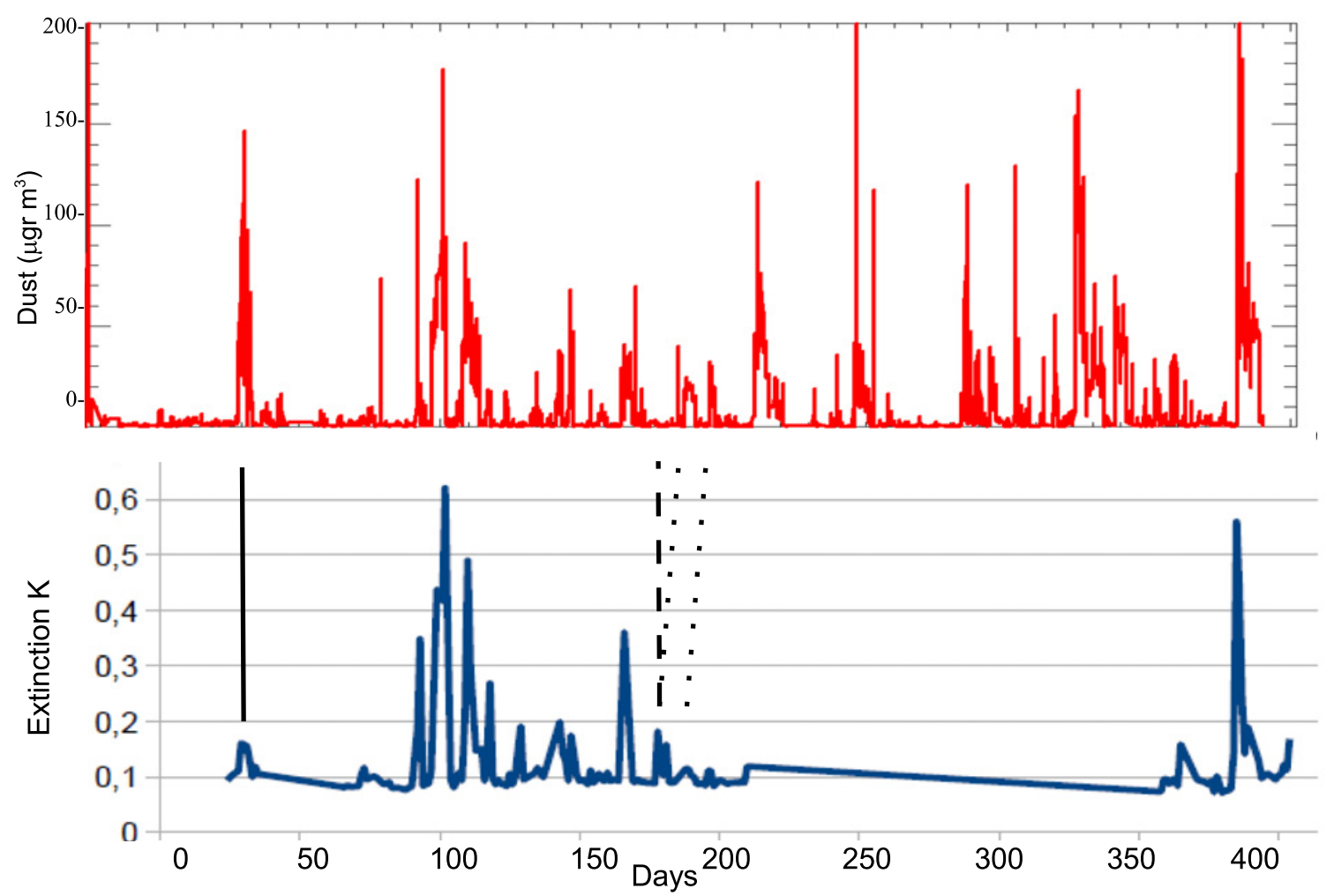

Figure 4. A comparison between the dust concentration measured with the particle counter (above) and the extinction along the line of sight measured at Carlsberg Telescope (below). While from the position of most of the peaks it can be seen that the behavior of both monitoring systems is similar for dust events, there are some discrepancies that show the not uniform distribution of the dust. A local layer could produce high concentration but the extinction is low (first peak on the left, marked with a continuous line); the opposite is seen in case of a high altitude thick layer, where we would measure low levels of dust concentration while extinction could be considerable (dashed line); one could also argue that in case of a high altitude layer there must be a time delay due to the settling of dust, and the extinction measured earlier could only be recorded as dust in a later moment (shown with two fine dotted lines in the middle of the plot).

smaller systems which complements the set of instruments previously described. We thus have a Sky Quality Meter to measure the brightness of the sky during the night, in particular to seek the effect of light pollution; a Lightning Detector to warn about approaching storms potentially dangerous for the electronic systems of the telescope; an All-Sky Camera for clouds detection. We also plan to mount a photometer to measure atmospheric extinction especially during dust storms.

\subsection{Sky Quality Meter}

The last 20 years have seen a moderate industrial and touristic development in La Palma and the number of inhabitants has been kept at about 90000 persons. But even if La Palma is a small island the fear of increasing light pollution is always present. Since 1992 in Canary Islands there is a Sky Quality Law and a dedicated office in charge to regularly monitor and control the rate of light pollution. The first published measurements of Night Sky Background (NSB) levels from the Roque de Los Muchachos Observatory dates to 1998 [2]. Few years later, in 2004, at the TNG we made similar measurements of light pollution from scientific spectra [23]. It can be seen a low level of light pollution (due to $\mathrm{Na}$ lamps) up to 0.05-0.1 mag. in the $\mathrm{V}$ band and, with respect to the measurements of 1998 by Benn and Ellison [2], a reduction of about $50 \%$ in the $\mathrm{Hg}$ polluting lines as an effect of the application of the Canary Sky Law. In 2006 we bought the Sky Quality Meter (SQM, from Unihedron [32]), an hand-held system with $80 \mathrm{deg}$ acceptance angle which (when used) typically measured a NSB of 21.6-21.8 mag/arcsec ${ }^{2}$ in the $\mathrm{V}$ band. The measurements are available at the Unihedron web page. Since the measurements campaigns were depending on the curiosity and willingness of someone to get outside and hold the SQM pointing at zenith during the measurements, we decided in 2013 to upgrade to the SQM-LE. The improvements with the new device are the Ethernet connection, that allows to obtain measurements remotely and at regular time spans, and secondly the acceptance angle reduced to $20 \mathrm{deg}$, that allows to exclude the effect of light coming from air glow (and in several occasions from the Milky Way) over the measurements.

\subsection{Lightning detector}

Another gadget that we decided to include in our set of instruments for atmospheric monitoring is the lightning detector LD-250 from Boltek [29]. Risk of lightning on the mountain is high and the fundamental purpose of this instrument is to determine the size and speed of the approaching thunderstorm in order to switch everything off 


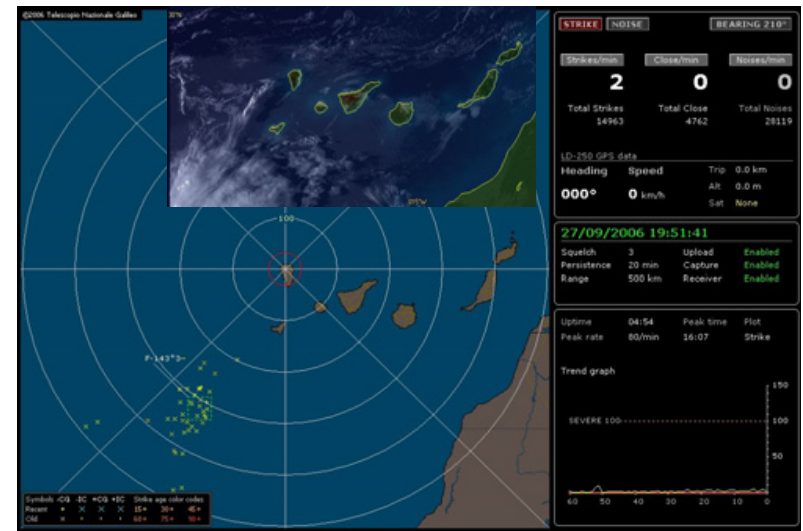

Figure 5. A comparison between the map provided with the LD-250 lightning detector, where detected lightning are represented as crosses $(x)$, and the satellite image of the approaching thunderstorm (in the inset).

before an electric discharge could affect the electronics of the telescope. Even if the dome of the TNG is completely metallic and creates a wonderful Faraday cage there still are risks of damage to the electronic systems of the telescope from the ground connection, from the main power supply and from the telephone lines. Due to this, the criteria we adopt in case of approaching thunderstorms is to disconnect most of the computers and systems from the main supply. We think it is better to spend some time in rebooting the whole system than to wait for spare parts (when available). The LD-250 has a range of $500 \mathrm{~km}$ and a graphical representation with the map centered at the system location, as in Fig. 5.

\subsection{All Sky Camera}

Still with the purpose of increasing safety of the telescope and the optimization of the observations, during the summer of 2014 we have installed an All Sky Camera (2ПSky based on a SBIG 340) with dedicated hardware and software from Cilium Engineering [30]. We mounted the camera at the top of the DIMM tower and the Telescope Operator or the Astronomer are warned about the percentage and direction of cloud coverage (as in Fig. 6): thanks to this feature it is possible to optimize the observations by choosing the scientific targets in a portion of the sky which is clear of clouds or even proceed to close the dome when rain drops get to the optics. It has often happened in fact to get rain over the main mirror of the telescope even while the RH sensor has been measuring $50 \%$ or less of humidity during the whole night. The rain is probably due to high layers of cirrus and the effect is only seen the day after in form of many tiny spots (or even trails) over the optical surface of the mirror. With the 2ПSky we hope to detect the cirrus layers in time and, if not, at least to see the rain drops over the lens of the camera and close the dome before the mirror gets too dirty.

\subsection{Photometer}

Since 2013 the Carlsberg Meridian Circle Telescope at the Roque de Los Muchachos Observatory is not measuring

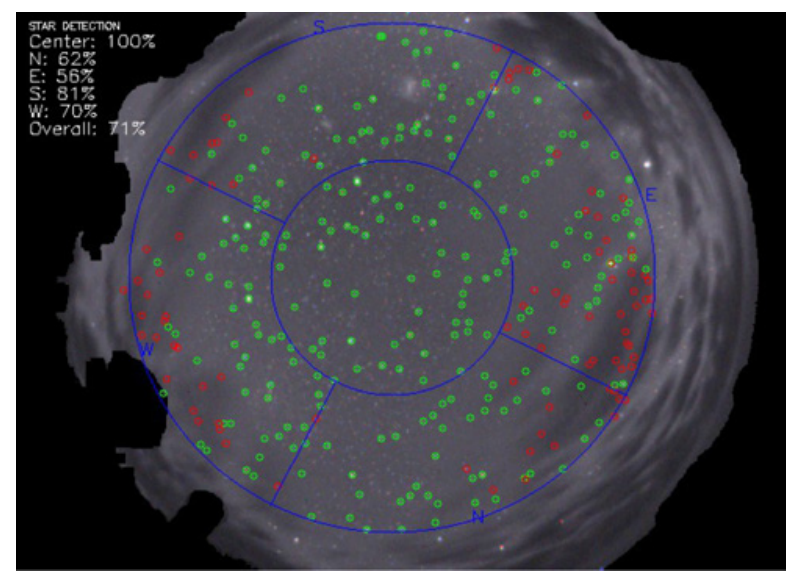

Figure 6. A picture from the $2 \Pi$ Sky where it can be seen that the whole sky is divided into 5 sectors into which colored circles represent detected or not-detected (due to clouds) stars.

extinction anymore. As a consequence the next upgrade we are preparing is a photometer to be piggy-back mounted on the telescope tube of the DIMM. This photometer is composed of a 4" Maksutov, a SBIG ST2000XMI camera and a set of 5 filters (UBVRI) in order to be able to measure NSB and extinction. We already have the pieces and we presume to set up the complete system before the end of this year. We plan to automatize the system and make publicly available the data of the atmospheric extinction as it has been for nearly 30 years with the Carlsberg Telescope.

\section{Conclusions}

From this simple review it can be seen that at TNG we monitor a wide range of atmospheric parameters with the main purposes of the (1) safety of the telescope and (2) the optimization of the observations. For the first of these purposes the data from the weather stations, lightning, clouds and dust must be available on the web page almost in real time. The optimization of telescope operations could be in real time as well (depending essentially on the observing program, but also on the Astronomer or Telescope Operator response time, for example as far as cloud coverage, seeing or extinction values are concerned) or after a more elaborate processing of the data (image quality assessment, tuning of the air conditioning, active optics look up tables, PID tuning of the tracking system, etc.). A side benefit of this is the archiving of data which are stored continuously and can be analyzed in a later moment (and remotely) in order to characterize the site and find important relationships between atmospheric parameters (for example through the principal component analysis, again [9]). The correlations that could appear would be useful at the moment of forecasting the local behavior of the weather or the seeing and easily adapt the scientific observations or the telescope setup following the actual conditions. Another important aspect of the atmospheric monitoring at the TNG is that the measured parameters are always available online to the other institutions. Even if the Roque de Los Muchachos 
Observatory is characterized by a complex orography which produces micro-climates and what is measured at one site is not necessarily the same in another site only a few hundreds of meters away, we try to be useful to the other institutions as much as their atmospheric monitoring systems are useful to us.

We would like to thanks an anonymous referee who gave useful suggestions that helped us on focusing the subject of this paper.

\section{References}

[1] Barbieri Cesare, SPIE 2871, 244-255 (1997)

[2] Benn Chris, New Astronomy Reviews 42, 503-507 (1998)

[3] Cavazzani S, MNRAS 411, 1271-1283 (2011)

[4] Cavazzani S, MNRAS 419, 3081-3091 (2012)

[5] Cavazzani S, MNRAS 429, 1849-1857 (2013)

[6] Cosentino Rosario, SPIE 8446 (2012)

[7] Della Valle Antonio, MNRAS 401, 1904-1916 (2010)

[8] Garcia de Gurtubai Albar, SPIE 8444 (2012)

[9] Garcia de Gurtubai Albar, MNRAS 429, 506-515 (2013)

[10] Garcia-Gil Alejandro, PASP 122, 1109-1121 (2010)

[11] Ghedina Adriano, SPIE 5489, 227-234 (2004)

[12] Guerrero M.A., New Astronomy Reviews 42, 529532 (1998)
[13] Lombardi Gianluca, PASP 118, 1198-1204 (2006)

[14] Lombardi Gianluca, PASP 119, 292-302 (2007)

[15] Lombardi Gianluca, SPIE 7012 (2008)

[16] Lombardi Gianluca, A\&A 483, 651-659 (2008)

[17] Lombardi Gianluca, MNRAS 399, 783-793 (2009)

[18] Lombardi Gianluca, SPIE 7733 (2010)

[19] Lombardi Gianluca, MNRAS 416, 1585-1590 (2011)

[20] Mancini Dario, New Astronomy Reviews 42, 425-429 (1998)

[21] Mancini Dario, MemSAIt 64, 675 (1993)

[22] Muñoz-Tuñon Casiana, New Astronomy Reviews 42, 409-416 (1998)

[23] Pedani Marco, New Astronomy 9, 641-650 (2004)

[24] Porceddu Ignazio, New Astronomy Reviews 42 , 447-449 (1998)

[25] Porceddu Ignazio, Astronomical Site Evaluation in the Visible and Radio Range. ASP Conference Proceedings (J. Vernin, Z. Benkhaldoun, and C. Muñoz-Tuñón. San Francisco, Astronomical Society of the Pacific, vol. 266, p. 432, 2002)

[26] Porceddu Ignacio, SPIE 4844, 358-365 (2002)

[27] Racine René, PASP 103, 1020-1032 (1991)

[28] Sarazin Marc, A\&A 227, 294 (1990)

[29] http://www.boltek.com/product/ld250-longrange-detection-kit

[30] http://www.cilium.pl

[31] http://www.tng.iac.es/weather

[32] http://unihedron.com/index.php 